\title{
Cooperative Spectrum Sensing for Cognitive Radio-Wireless Sensors Network Based on OR Rule Decision to Enhance Energy Consumption in Greenhouses
}

\author{
Haythem Alhumud ${ }^{1,2}$, Mohammed Zohdy ${ }^{1}$, Debatosh Debnath ${ }^{3}$, Richard Olawoyin ${ }^{4}$, \\ Sayed Ali Arefifar ${ }^{1}$
}

\author{
${ }^{1}$ Electrical and Computer Engineering Department, Oakland University, Rochester Hills, MI, USA \\ ${ }^{2}$ Ministry of Rural Affairs and Municipality, Riyadh, KSA \\ ${ }^{3}$ Computer Science and Informatics Department, Oakland University, Rochester Hills, MI, USA \\ ${ }^{4}$ Industrial and Systems Engineering Department, Oakland University, Rochester Hills, MI, USA \\ Email: halhumud@Oakland. edu
}

How to cite this paper: Alhumud, H., Zohdy, M., Debnath, D., Olawoyin, R. and Arefifar, S.A. (2019) Cooperative Spectrum Sensing for Cognitive Radio-Wireless Sensors Network Based on OR Rule Decision to Enhance Energy Consumption in Greenhouses. Wireless Sensor Network, 11, $1-11$.

https://doi.org/10.4236/wsn.2019.111001

Received: January 14, 2019

Accepted: January 28, 2019

Published: January 31, 2019

Copyright $\odot 2019$ by author(s) and Scientific Research Publishing Inc. This work is licensed under the Creative Commons Attribution International License (CC BY 4.0).

http://creativecommons.org/licenses/by/4.0/

\section{(c) (i) Open Access}

\begin{abstract}
Wireless sensors networks (WSNs) combined with cognitive radio have developed and solved the limited space of the frequency spectrum. In this paper, we propose different types of spectrums sensing and their own decisions depend on the probabilities that applied into fusion center, and how these probabilities' techniques help to enhance the energy consumption of WSNs. In the same way, the importance of designing balanced distribution between the wireless sensors networks and their own sinks. This research also provides an overview of security issues in CR-WSN, especially in Spectrum Sensing Data Falsification (SSDF) attacks that enforces harmful effects on spectrum sensing and spectrum sharing. We adopt OR rule as four types of CRSN sensing protocolin greenhouses application by using Matlab and Netsim simulators. Our results show that the designing balanced wireless sensors and their sinks in greenhouses are very significant to decrease the energy, which is due to the traffic congestion in the sink range area. Furthermore, by applying OR rule has enhanced the energy consumption, and improved the sensors network lifetime compared to cognitive radio network.
\end{abstract}

\section{Keywords}

Wireless Sensors Networks, Cognitive Radio Networks, Spectrum Decision, Greenhouses, Cooperative Sensing, Greenhouse, Energy Efficient Based on Spectrum Decision 


\section{Introduction}

Wireless sensor networks (WSNs) are one of the communication systems that have been recently adopted by technological advancements of the modern world. This type of communication has greatly helped several people to share data with others in different geographic locations. Conversely, sending and receiving information through WSNs has risen which may cause the spectrum scarcity problem due to some limitations of the available frequencies regulation that conducted by Federal Communication Commission (FCC) [1]. FCC controls the radio spectrum and its availability and giving the authorization to the primary user to use the spectrum. Using some methods, such as cognitive radio based in wireless sensors networks (CR-WSNs), can overcome the spectrum scarcity. Cognitive Radio system generally has four components: Primary Users (PUs), Base station for the PUs, Cognitive Radio Users or Secondary Users (SUs), and Base station for the SUs. The PUs as the name implies, are the main users who have license as such are authorized to use the channel spectrum by the PU-base station. Whereas, the SUs are not regular users as such must request channel spectrum from the CR-base station. Cognitive radio allows the communication devices to use some of the unoccupied frequencies in white space (unused space) without any interference to other users that use occupied frequencies in black space (used space). There are some characteristics in CR-WSNs, one of which is management spectrum which consists of four main fundamental components to allow wireless sensor networks to dynamically access the available channels and benefits from the opportunistic channels: 1) Spectrum sensing, 2) Spectrum Decision, 3) Spectrum Sharing and 4) Spectrum Mobility. In this research, large-scale implementation of the fully observed greenhouses attracts the investors these days. However, large-scale implementation of wireless sensor networks WSNs in greenhouses rise with the spectrum scarcity problem. But by applying cognitive radio in wireless sensors networks, overcome the limitation of the covering wide-ranging fields [2]. In this paper, we discuss energy efficient based in spectrum sensing and OR technique to increase the network life time, in addition to an overview of security issues in cognitive radio system.

\section{Cooperative Spectrum Sensing for Cognitive Radio-Wireless Sensors Network}

Spectrum sensing is considered one of the most essential methods of CR-WSNs. This feature allows sensors to sense, analyze, and monitor parameters of the radio channel that are used for communication without causing harmful interference that may happen between these sensors. There are some factors cognitive radio system must accommodate and consider to perform sufficiently:

- Transmission Recognition: cognitive radio should be able to sense and determine the type of transmission that is detected, especially for the primary users to avoid any interventions that may be caused.

- Regularly spectrum sensing: necessary for the cognitive radio system to regularly sense the spectrum to observe the primary user arrival. 
- Alternative spectrum: during the arrival of the primary user to occupy the spectrum, the cognitive radio system must have an alternative spectrum available to substitute the channels.

Spectrum Decision is the approach to conduct the best available spectrum which can examine the spectrum if it is qualified and meet the secondary user's requirements and decided that spectrum is ready to be occupied by SUs and free of any conflict may be caused with the (PUs).

Spectrum Sharing in this method, it can control the area of spectrum in case of having many cognitive radio users that attempt to access in the spectrum and manage it to avoid any interference between PUs and SUs.

Spectrum Mobility the basic concept of spectrum mobility is when the SUs are occupied in some holes of the spectrum during the Primary user's arrival, it can move on the SUs to another available unoccupied place to ensure there is no interruption during this movement. In cognitive radio, there are two sensing techniques: 1) Non-cooperative sensing and 2) Cooperative sensing.

- Non-cooperative spectrum sensing, this kind of spectrum sensing happens when each cognitive radio user performs by itself which is independently not relying on other users.

- Cooperative spectrum sensing, this method of spectrum sensing is conducted by some multiple numbers of various radios in the cognitive radio network. Specifically, the fusion center (FC) will gather information of the signals from different radios through the node in the network and check, modify and accommodate all the cognitive radio network to be fully set. The key advantage of cooperative sensing is it can be used to prevent the multipath fading and interference of the SUs with licensed users PUs. In Cooperative spectrum sensing, we can use binary hypothesis testing to predict the presence and the absence of the signals as shown below:

$$
x(n)=\left\{\begin{array}{l}
w(n) H_{0} \text { available for } S U s \\
h * s(n)+w(n) H_{1} \text { currently Occupied by } P U s
\end{array}\right.
$$

where $s(n)$ is the transmitted signals for the primary user, $w(n)$ is the Additive White Gaussian Noise (AWGN) of variance $\sigma^{2}$ and $h$ is the amplitude gain of the channel. OR Rule Decision in Cognitive Radio-Wireless Sensors Network. The fusion techniques in cooperative spectrum sensing can be classified into soft and hard decisions schemes [3], as shown in Figure 1.

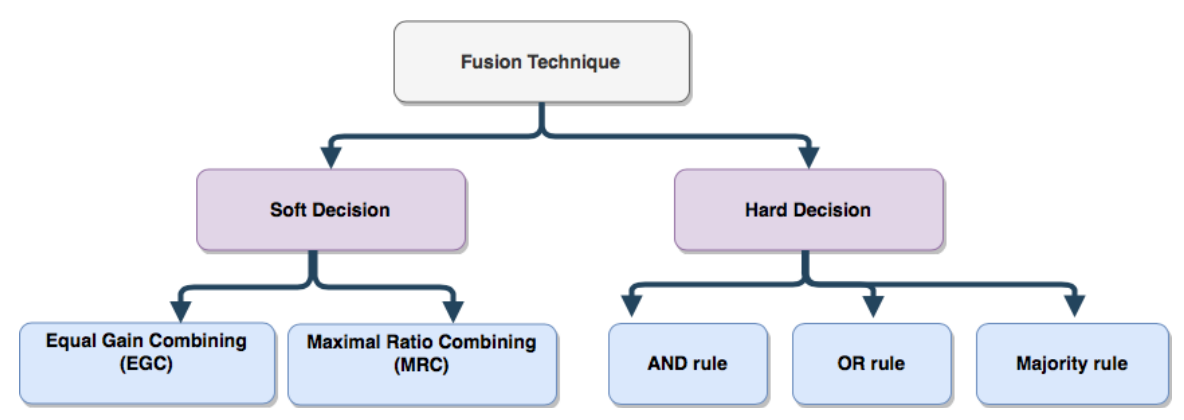

Figure 1. Fusion techniques in cooperative spectrum. 
The decision between the two hypotheses $H_{0}$ or $H_{1}$ in OR rule decisions explained in the table as shown below:

\begin{tabular}{ccccc}
\hline & Hypotheses & \multicolumn{2}{c}{ The Status } & The OR rule decisions \\
\hline 1 & $\left(H_{0} ; H_{0}\right)$ & Decide $H_{0}$ when $H_{0}$ is true & Probability of No Detection \\
2 & $\left(H_{0} ; H_{1}\right)$ & Decide $H_{0}$ when $H_{1}$ is true & Probability of Missed Detection $\left(P_{M D}\right)$ \\
3 & $\left(H_{1} ; H_{0}\right)$ & Decide $H_{1}$ when $H_{0}$ is true & Probability of False Alarm $\left(P_{F A}\right)$ \\
4 & $\left(H_{1} ; H_{1}\right)$ & Decide $H_{1}$ when $H_{1}$ is true & Probability of Detection $\left(P_{D}\right)$ \\
\hline
\end{tabular}

Probabilities of miss detection and false alarm based in spectrum sensing of cognitive radio systems are essential to enhance the energy consumption of wireless sensor nodes in cognitive radio domain, also they help to decide the status of the spectrum, either it is occupied/busy or it is available and free to be taken.

Our goal in this research, is increasing the probability of all channels by allowing SUs to pick the channel subsets, these subsets size can make an arrangement between the energy decreasing and the enhancement in the probability of miss-detection (MD). The cooperative slotted target has fixed decision delay to allow the sensor node as the secondary users in sleep mode during cooperation and in idle mode to be ready for receiving data signals depending on its channel map (CM) which is a sequence. Moreover, in case of the secondary user transmitting data packet to one specific channel and in the same time prevents other SUs to sense the same channel to keep saving the sensors node energy and decreasing the entire delay [4].

The probability of detection can be computed by the equation shown below

$$
P_{D}=Q_{(x)} \frac{\beta_{E D}-\left(\sigma_{s}^{2}+\sigma_{n}^{2}\right)}{\left(\sigma_{s}^{2}+\sigma_{n}^{2}\right) / \sqrt{N}}
$$

The probability of miss detection can be computed by the equation shown below

$$
P_{M D}=Q_{(x)} \frac{\sqrt{N}\left(\beta_{E D}-\left(\sigma_{s}^{2}+\sigma_{n}^{2}\right)\right)}{\left(\sigma_{s}^{2}+\sigma_{n}^{2}\right)}
$$

The probability of false alarm can be computed by,

$$
P_{F A}=Q_{(x)} \frac{\sqrt{N}\left(\beta_{E D}-\sigma_{n}^{2}\right)}{\left(\sigma_{n}^{2}\right)}
$$

where $N$ is the number of signal samples, $\beta_{E D}$ is the threshold, $\sigma_{s}^{2}$ is the signal power, $\sigma_{n}^{2}$ is the noise power derivation and $Q_{(x)}$ is the Generalized Marcum function $\frac{1}{\sqrt{2 \pi}} \int_{t}^{\infty} e^{-\frac{u^{2}}{2}} \mathrm{~d} u$.

Channel sensing can be recognized by energy detection, that is known as a method to detect the signals either Pus signal if the signal power is above the threshold or SUs signal if the signal power is below the threshold [5], if the signals detected as secondary user then all the consequence information of the sensing is gathered and analyzed at a FC to generate a decision by using OR rule on the channel occupancy. 
OR rule means if at least one N SU detects the primary users that are occupied, then final decision confirms that there is a primary user in use of the channel. The OR-rule technique is an excellent choice for cooperative spectrum sensing in cognitive radio networks compared to other techniques and it can be applied as:

$$
\begin{gathered}
Q_{\left(P_{F A}-O R \text { rule }\right)}=1-\left(1-P_{F A}\right)^{N} \\
Q_{\left(P_{D}-O R \text { rule }\right)}=1-\left(1-P_{D}\right)^{N}
\end{gathered}
$$

As shown in Figure 2 and the flow chart in Figure 3, the localization of WSNs can be affected positively or negatively on sensors network lifetime depending on the design of the wireless sensors and their sinks for instance, centralized approach or distributed approach. The area around sinks is crowded, that is due to heavy traffic congestion of the communication between the sensors in sinks range area which ended up to consume excessively high energy consumption [6], so as a result limitation of the network lifetime in WSNs.

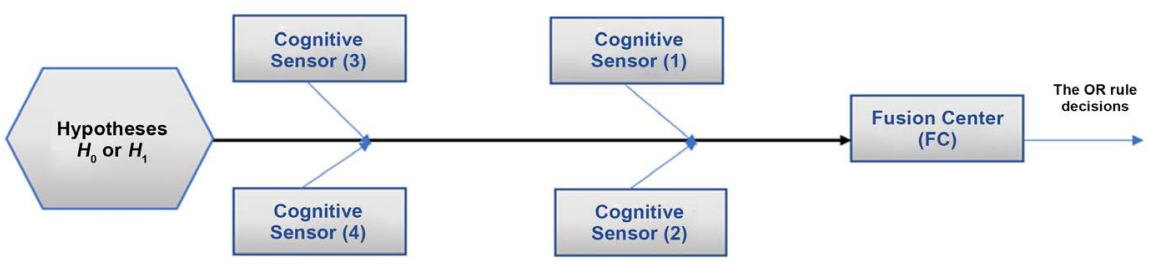

Figure 2. Explanation of the process of OR rule decisions.

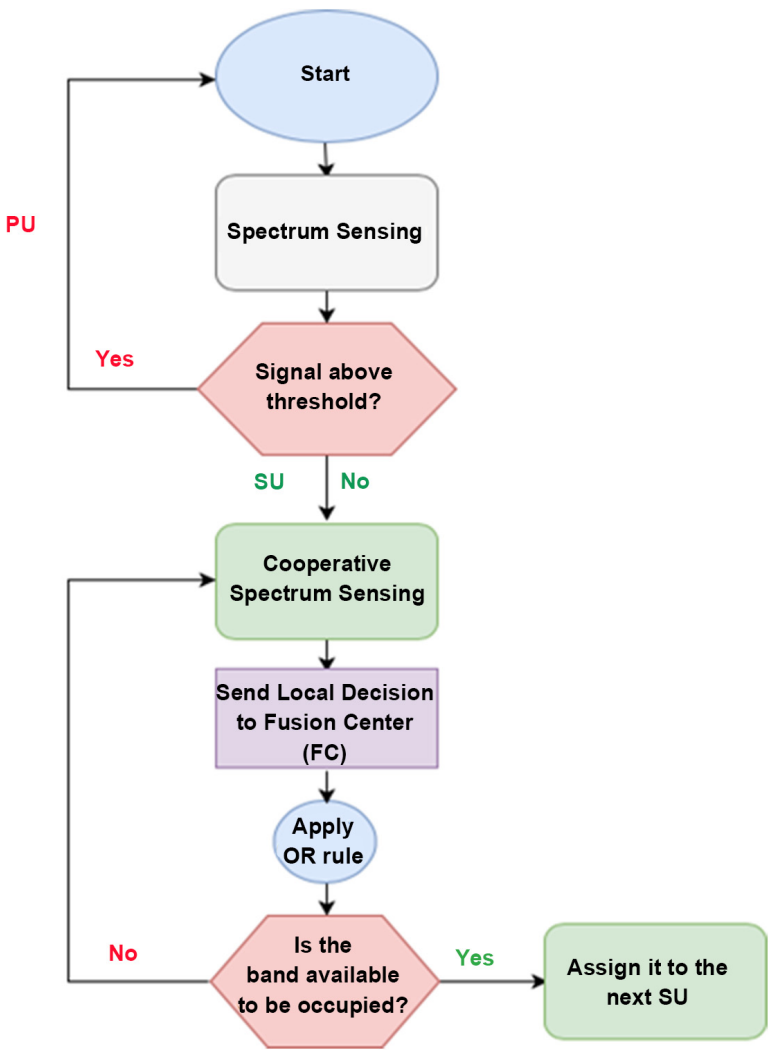

Figure 3. Flow chart explains the process of OR rule decisions. 


\section{Security Issues in Cognitive Radio}

CR-WSNs is more challenging than in traditional WSNs. This is due to the limitations of WSNs; low battery power, storage, communications speed, and computational capability. These limitations open doors to security challenges of the use of CR-WSNs, among other challenges.

CR-WSNs suffer from similar security issues existing in the traditional wireless sensor networks, such as Physical security issues. Because sensors are mostly left idle, they are liable to be physically manipulated to steal data going in and out of it, or they can be tempered with or destroyed. In a typical Physical layer attack, the attacker interferes and disrupts the communications between CR wireless sensors by jamming the radio signals being transmitted on the wireless channel [7]. Attackers also target the upper layers; the MAC layer and the Network/Routing layer to perpetrate attacks such as Denial of Service (DoS), Sybil, and Wormhole. In addition to these security challenges similar to those of the traditional wireless sensor network, CR-WSNs are vulnerable to other security threats, such as the lack of proper coordination between PUs and SUs. This lack of proper coordination between these two entities may result in data being exchanged, to be stolen, modified or injected with some false data by unauthorized users. Attackers can also use a technique called Spectrum Sensing Data Falsification(SSDF) [8] [9] to interfere with the channel spectrum used by the PUs to appear to be in use or on the other hand, not in use, so as to prevent the SUs from using it or cause Denial of Service (DoS), among other threats. Typically, the CR-Base station collects sensing information from the SUs about channel spectrums that are not being used by the PUs and make a prudent decision about the spectrums' availability, and then assign them to the SUs. In SSDF attack, one or more malicious or compromised SUs send(s) false sensing information to the CR-Base station in an effort to maliciously influence its decision to assign SUs perceived free channel spectrums under erroneous judgement as shown in Figure 4. This could lead to threats such as DoS. On the other hand, SSDF attack could lead to channel spectrum underutilization and hence reduce the overall performance of the network.

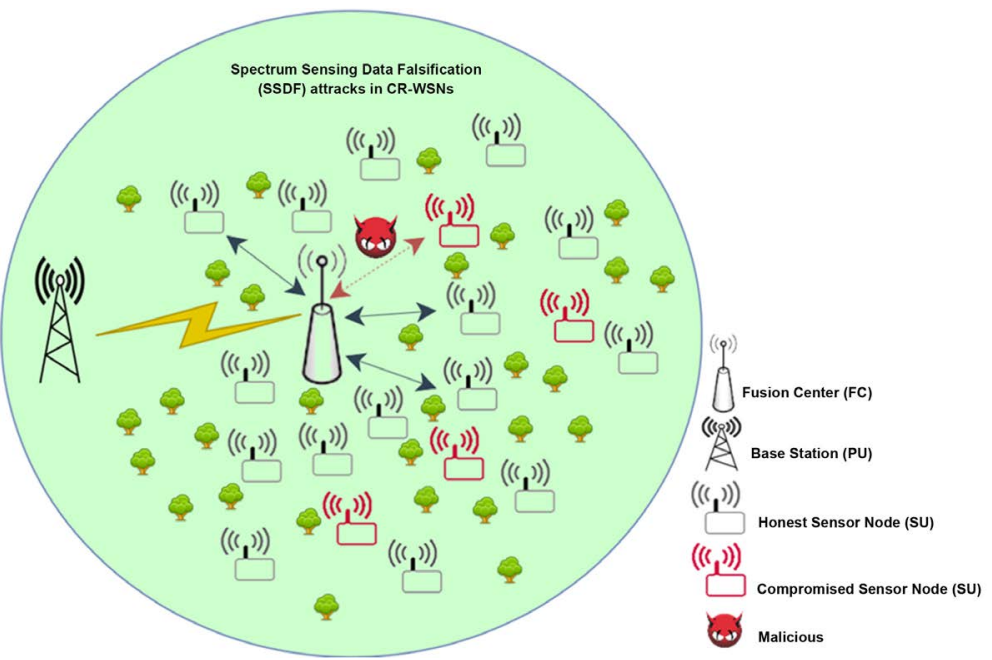

Figure 4. Explanation of SSDF attack in cognitive radio-wireless sensors network. 


\section{Results and Discussion}

\subsection{Experimental Setup}

We evaluate the performance of the proposed schemes using Matlab and Net Simsimulators. We consider 20 Greenhouses in a network and four CRSN types for temperature, humidity, $\mathrm{CO}_{2}$ and soil composed of 6 nodes/type/greenhouse. We assume that the power profile follows that of a typical Atheros Wi-Fi [10]. Transmission is based on CSMA/CA contention-based access. All channels undergo typical Rayleigh fading. Table 1 summarizes the simulation parameters. These parameters are selected based on similar previous works in [11] [12] but with some modifications, such as, the numbers of greenhouses that applied into the simulators, total number of nodes, size of data packets, size of network area and balanced distribution of wireless sensors network in the greenhouses instead of random distribution on previous works.

Table 1. Simulation parameters.

\begin{tabular}{|c|c|}
\hline Definition & Value \\
\hline Number of Greenhouses & 20 \\
\hline Number of CRSN types & 4 \\
\hline Number of nodes/type & 6 \\
\hline Total number of nodes & 480 \\
\hline Network Area & $500 \mathrm{~m} \times 500 \mathrm{~m}$ \\
\hline Data Packet & 90 Kbyte \\
\hline$T_{X}$ power & $20 \mathrm{dBm}$ \\
\hline Noise Floor & $-70 \mathrm{dBm}$ \\
\hline Detection Threshold & $-20 \mathrm{dBm}$ \\
\hline Sensing Time & 2 slots \\
\hline
\end{tabular}




\subsection{Energy Consumption}

We compare the results between CR-WSN and the OR rule applied in CR-WSN. The CRSN energy consumption per node in a single day ranges from 14 to 30 $\mathrm{mJ}$. The OR rule technique energy consumption per node in a single day ranges from 8 to $16 \mathrm{~mJ}$. As shown in Figure 5, OR rule technique consumes less energy consumed that is due to low packet transmission rate, collisions, retransmissions and the avoidance of putting sensors in the sinks range area since they balanced distribution.

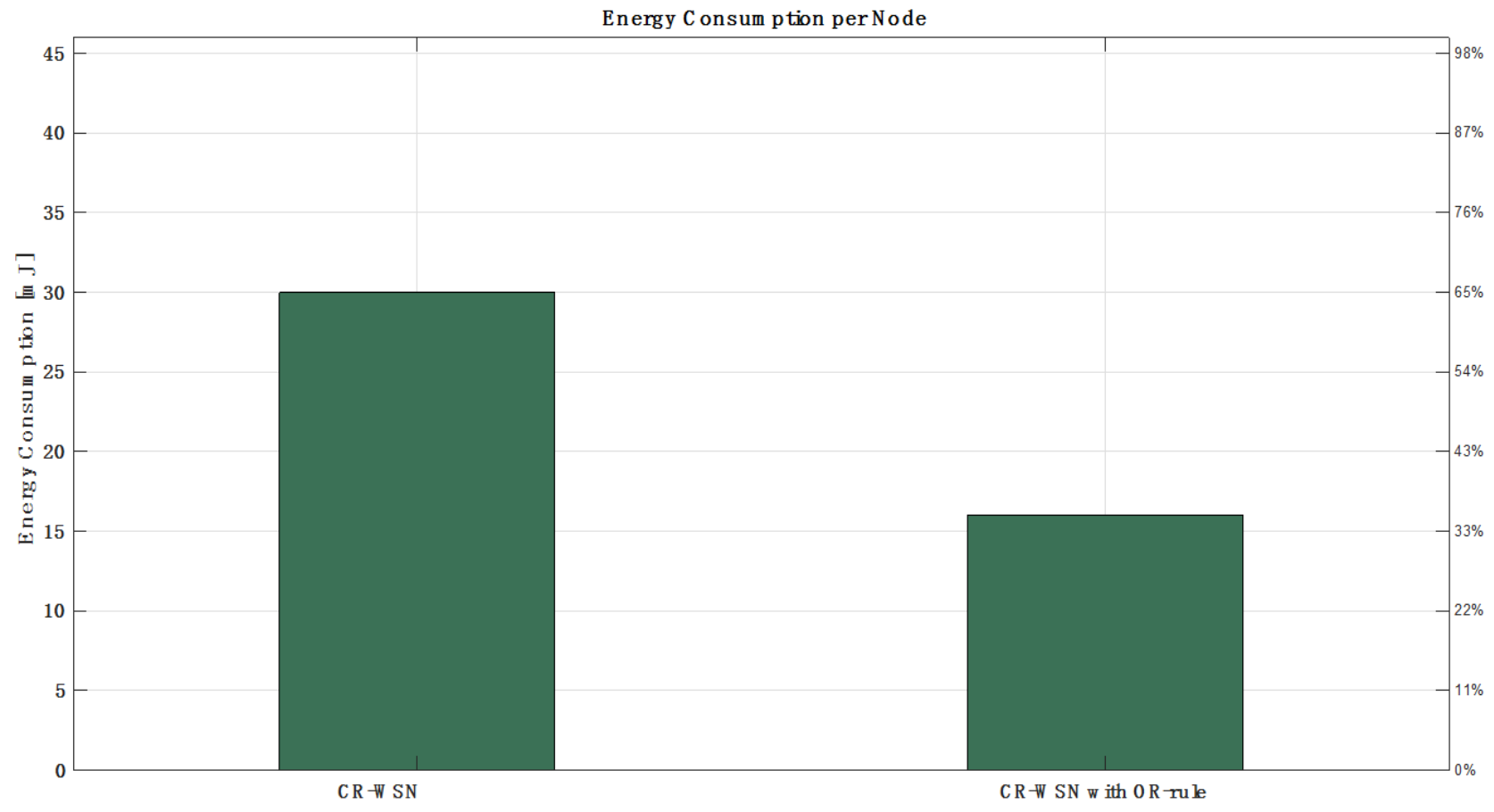

Figure 5. Energy consumption per node.

\subsection{Network Lifetime}

After a period of time $\mathrm{T}$, some nodes drain their batteries and they are treated as dead nodes. In this section, we present the comparison of CR-WSN and CR-WSN that uses OR rule. Figure 6 and Figure 7 show the CR-WSNs nodes and CR-WSNs with OR rule nodes after the time period T. In Figure 6, in CR-WSNs too many nodes are dead due to the CR energy-hungry techniques. Meanwhile, Figure 7 that shows OR-rule protocol nodes have low energy consumption, CR-WSN nodes that use OR rule are better and still operating excellent condition and only few of them are dead.

The network lifetime can be observed for a time period of 38 months for the CR-WSNs and CR-WSNs with OR rule applied as shown in Figure 8. The existing CRSN techniques drain the node's battery fast and some sensors network died in 26 months, while the OR rule network is still alive for more than 38 months with excellent condition. 


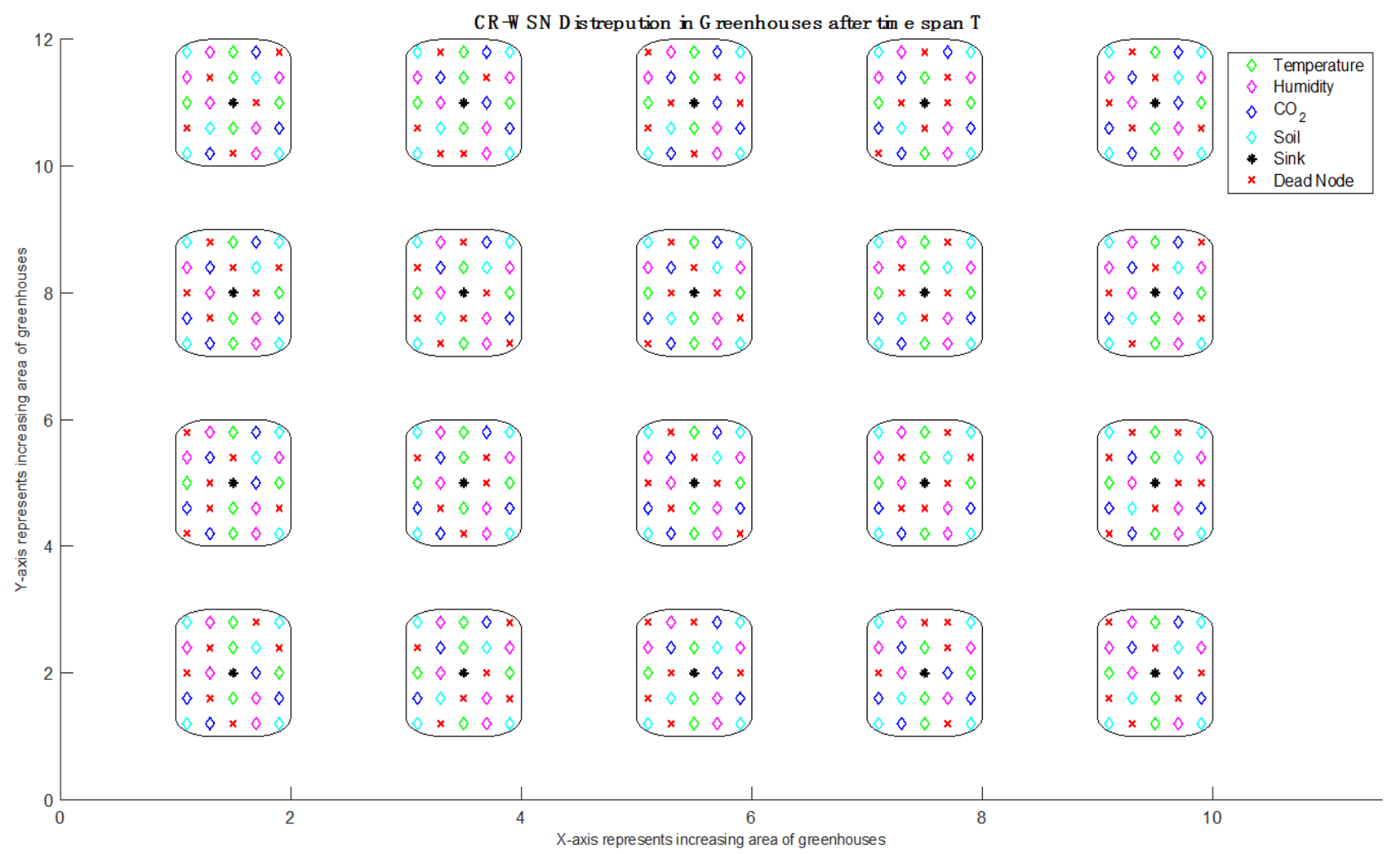

Figure 6. CR-WSN sensors after a period T (too many nodes are dead).

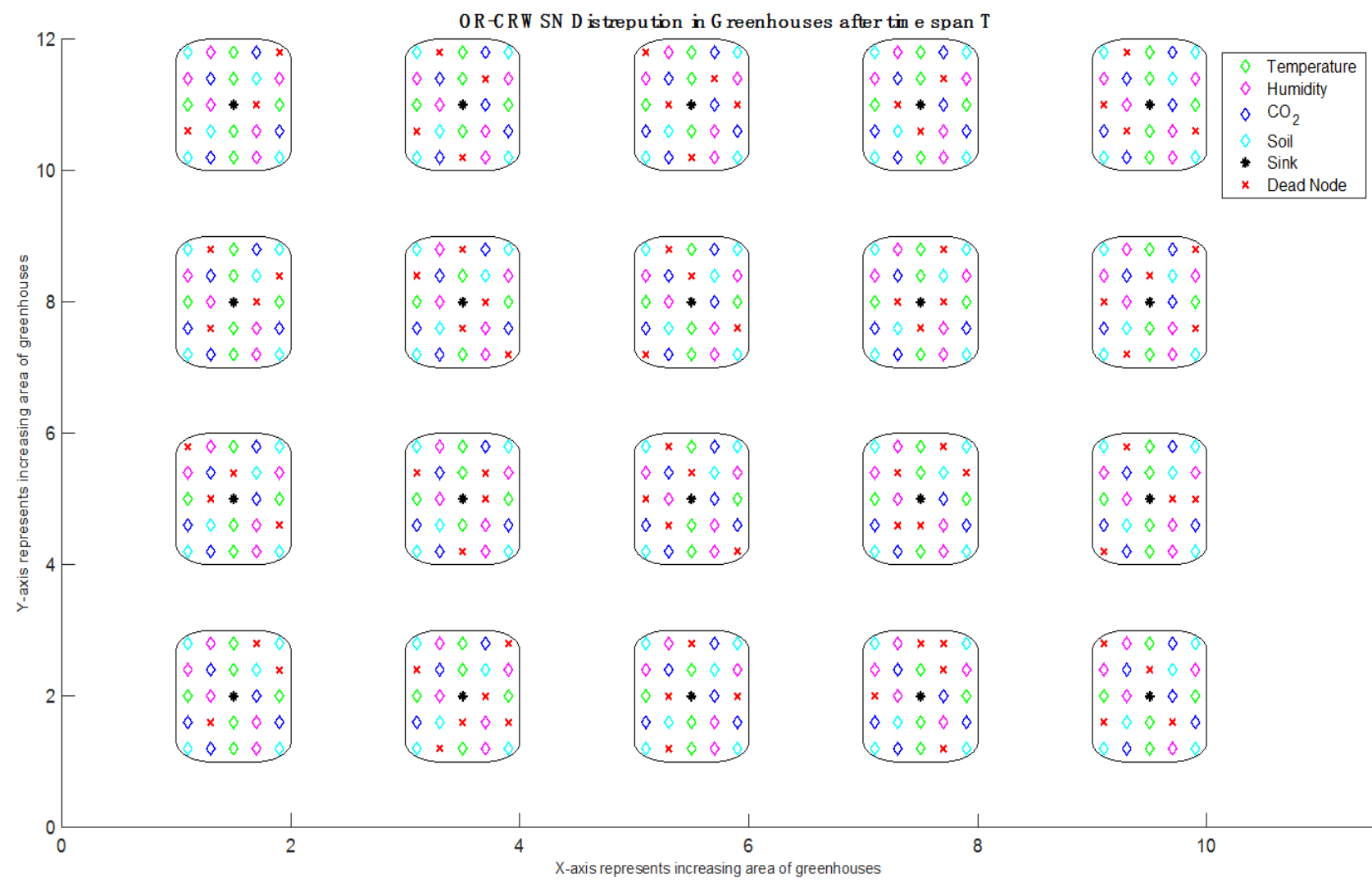

Figure 7. The OR-rule in CR-WSN sensors after a period T (operating in an excellent condition). 


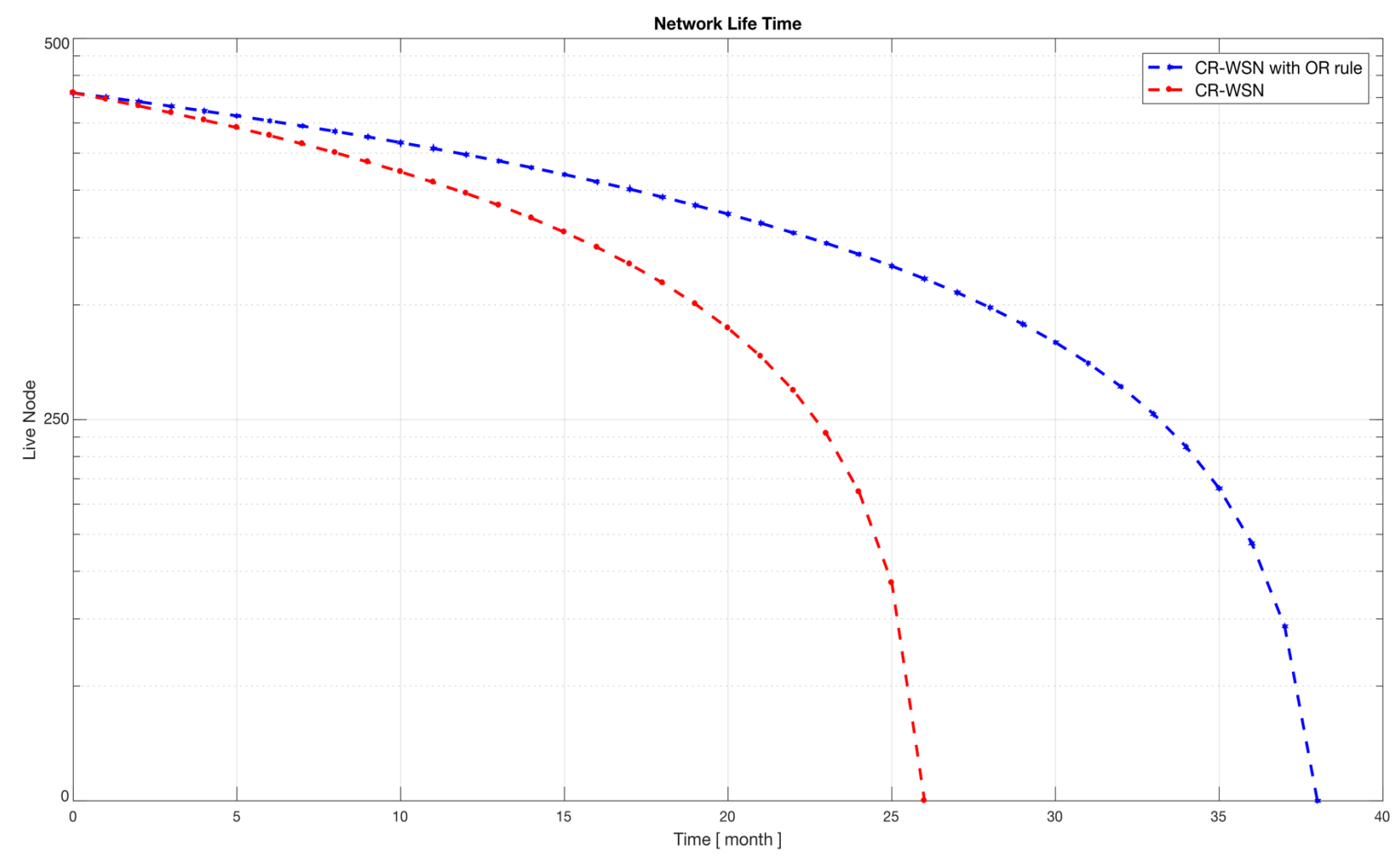

Figure 8. Network lifetime.

\section{Conclusion}

In this paper, we proposed an overview of security issues in cognitive radio system and the capability of OR-rule technique, especially in greenhouses application. Unlocalized sensors nodes estimate their position from the base-station node which required much power due to traffic congestion between sinks and the sensors in range area of the sinks. The use of OR-rule is considered as energy saving protocol that enables the investors to have desire of fully observed greenhouses while maintaining the minimum energy consumption and extend the sensor network lifetime. The proposed OR-rule has better improvement rather than other rules, in addition OR rule does not only save the consumed energy but also reduce the required capabilities of the nodes to be more simple nodes. Our results show that OR-rule has less energy consumption and has a longer lifetime compared to CR-WSN.

\section{Conflicts of Interest}

The authors declare no conflicts of interest regarding the publication of this paper.

\section{References}

[1] Chandwani, N., Jain, A. and Vyavahare, P.D. (2015) Throughput Comparison for Cognitive Radio Network under Various Conditions of Primary User and Channel Noise Signals. Radio and Antenna Days of the Indian Ocean (RADIO), Belle Mare, 21-24 September 2015. 
[2] Jin, O., Qiao, Y., Liu, A. and Zhang, L. (2018) EESS: An Energy-Efficient Spectrum Sensing Method by Optimizing Spectrum Sensing Node in Cognitive Radio Sensor Networks. Wireless Communications and Mobile Computing, 2018, Article ID: 9469106.

[3] Meena, O.P. and Somkuwar, A. (2014) Comparative Analysis of Information Fusion Techniques for Cooperative Spectrum Sensing in Cognitive Radio Networks. Proceedings of International Conference on Recent Trends in Information, Telecommunication and Computing, ITC 2014.

[4] Ali, H., Khattab, A. and Fikri, M. (2016) Generalized Implicit Cooperation with Slotted Contention in Cognitive Radio Wireless Sensor Networks. Proceedings of IEEE International Conference on Selected Topics in Mobile \& Wireless Networking (MoWNeT), Cairo, 11-13 April 2016, 1-8.

[5] Prema, G. and Narmatha, D. (2016) Performance of Energy a Ware Cooperative Spectruim Sensing Algorithm in Cognitive Wireless Sensor Network. Online International Conference on Green Engineering and Technologies (IC-GET), Coimbatore, 19 November 2016.

[6] Gharaei, N., Abu Bakar, K., Mohd Hashim, S.Z., Hosseingholi Pourasl, A., Siraj, M. and Darwish, T. (2017) An Energy-Efficient Mobile Sink-Based Unequal Clustering Mechanism for WSNs. Sensors (Basel), 17, 1858. https://doi.org/10.3390/s17081858

[7] Joshi, G.P., Nam, S.Y. and Kim, S.W. (2013) Cognitive Radio Wireless Sensor Networks: Applications, Challenges and Research Trends. Sensors, 13, 11196-11228. https://doi.org/10.3390/s130911196

[8] Althunibat, S., Denise, B.J. and Granelli, F. (2016) Identification and Punishment Policies for Spectrum Sensing Data Falsification Attackers Using Delivery-Based Assessment. IEEE Transactions on Vehicular Technology, 65, 7308-7321.

https://doi.org/10.1109/TVT.2015.2497349

[9] Akyildiz, I.F., Lo, B.F. and Balakrishnan, R. (2011) Cooperative Spectrum Sensing in Cognitive Radio Networks: A Survey. Physical Communication, 4, 40-62. https://doi.org/10.1016/j.phycom.2010.12.003

[10] Baradkar, H. and Akojwar, S. (2014) Implementation of Energy Detection Method for Spectrum Sensing in Cognitive Radio Based Embedded Wireless Sensor Network Node. International Conference on Electronic Systems, Signal Processing and Computing Technologies, Nagpur, 9-11 January 2014.

https://doi.org/10.1109/ICESC.2014.92

[11] Alhumud, H. and Zohdy, M. (2018) Adopting the Novel Generalized Implicit-OR Sensing Protocol to Decrease the Energy Consumption of Wireless Sensors in Greenhouse. Proceedings of IEEE International Conference on Electrical, Electronics, Computers, Communication, Mechanical and Computing (EECCMC), Tamil Nadu.

[12] Alhumud, H. and Zohdy, M. (2018) Managing Energy Consumption of Wireless Sensors Networks in Multiple Greenhouses. Wireless Engineering and Technology, 9, 11-19. https://doi.org/10.4236/wet.2018.92002 